\title{
Prevalence of Enterococcus faecalis in saliva and filled root canals of teeth associated with apical periodontitis
}

\author{
Qian-Qian Wang ${ }^{1}$, Cheng-Fei Zhang ${ }^{2}$, Chun-Hung $\mathrm{Chu}^{2}$ and Xiao-Fei $\mathrm{Zhu}^{1}$ \\ To investigate the prevalence of Enterococcus faecalis in saliva and filled root canals of patients requiring endodontic retreatment for \\ apical periodontitis. Patients with apical periodontitis who were referred for endodontic retreatment were examined. The type and \\ quality of the restoration, symptoms, quality of obturation were recorded. During retreatment, an oral rinse sample and root canal \\ sample were cultured using brain-heart infusion agar and bile esculinazide agar to select for $E$. faecalis. The 16S rRNA technique was \\ used to identify $E$. faecalis. A total of 32 women and 22 men (mean age: 38 years; s.d.: 11 years) and 58 teeth were studied. The \\ prevalence of $E$. faecalis was $19 \%$ in the saliva and $38 \%$ in the root canals. The odds that root canals harbored $E$. faecalis were \\ increased if the saliva habored this bacterium (odds ratio $=9.7 ; 95 \%$ confidence interval $=1.8-51.6 ; P<0.05$ ). Teeth with \\ unsatisfactory root obturation had more cultivable bacterial species in root canals than teeth with satisfactory root obturation \\ $(P<0.05)$. E. faecalis is more common in root canals of teeth with apical periodontitis than in saliva. The prevalence of $E$. faecalis in \\ root canals is associated with the presence of $E$. faecalis in saliva.
}

International Journal of Oral Science (2012) 4, 19-23; doi:10.1038/ijos.2012.17; published online 16 March 2012

Keywords: apical periodontitis; endodontic treatment; Enterococcus faecalis; saliva; 16S rRNA

\section{INTRODUCTION}

Enterococci are common bacteria that inhabit the gastrointestinal tract, oral cavity, and vagina of humans and animals. Although enterococci were initially regarded as non-virulent, they are now recognized as one of the major causes of nosocomial infections worldwide. ${ }^{1}$ In dentistry, Enterococcus species, in particular Enterococcus faecalis, have been found to be associated with chronic periodontitis ${ }^{2}$ and failed root canal treatments involving chronic apical periodontitis. ${ }^{3}$ Furthermore, in a study of 36 patients, E. faecalis was commonly isolated from periapical lesions refractory to endodontic treatment. ${ }^{4}$

A widely held assumption is that microorganisms found in the root canal space are derived from those colonizing the oral cavity. ${ }^{5}$ However, it has recently become apparent that this hypothesis requires elaboration. Whereas E. faecalis was present in oral rinse samples from patients who had endodontic treatment, ${ }^{5}$ this bacterium was rarely detected in healthy mouths. ${ }^{6}$ Given the ubiquitous occurrence of enterococci in food products, Kampfer speculated that oral niches, such as untreated necrotic root canals, can become transiently colonized. ${ }^{7}$ The microenvironment of root canals may especially favor the survival of enterococci and the establishment of long-standing local infections. ${ }^{8}$ Other conditions, such as the quality of obturation, could conceivably also influence the colonization of E. faecalis and hence microflora in roots, either directly or indirectly.

Whether there were any differences between the presence of $E$. faecalis in saliva and in filled root canals is still unknown, and the difference between the presences in unsatisfactorily obturated canals and in well-obturated canals is also still unclear. In this study, we aimed to investigate the association between the prevalence of $E$. faecalis in saliva and in root canals of previously endodontically treated teeth that needed retreatment because of apical periodontitis. We also evaluated the association between the presence of $E$. faecalis in root canals and various clinical and physical variables, including the type and quality of restoration and the quality of obturation.

\section{MATERIALS AND METHODS}

\section{Patient recruitment}

Adult patients aged 18 years or older who attended the Peking University School and Hospital of Stomatology between September 2006 and December 2008 were invited to participate in this study if they showed radiological evidence of apical periodontitis based on the AAE accepted diagnostic recommendations ${ }^{9}$ and also requiring endodontic retreatment. Patients who smoked, were pregnant, or had diabetes or other systemic conditions were excluded, as were those who had undergone treatment with local or systemic antimicrobial agents within the previous 6 months and those with generalized periodontal disease with a pocket depth of $\geqslant 4 \mathrm{~mm}$. Also excluded were patients requiring retreatment owing to missing canals, separated endodontic instruments, perforations or calcified root canals in which the apex was inaccessible. The study was approved by the Ethics Committee of Peking University Institutional Review Board. Patients were informed of the study protocol and aims, and written consent was obtained before recruitment.

${ }^{1}$ Special Dental Department, School and Hospital of Stomatology, Peking University, Beijing, China and ${ }^{2}$ Faculty of Dentistry, The University of Hong Kong, Hong Kong SAR, China

Correspondence: Dr. CF Zhang, Faculty of Dentistry, The University of Hong Kong, Hong Kong SAR, China

E-mail: zchengfei@yahoo.com

Received 5 November 2011; Accepted 29 January 2012 


\section{Clinical assessment}

During the clinical examination and retreatment, the location of affected teeth, number of root canals per tooth, and clinical signs and symptoms (presence of pain, hypermobility, sinus tract and its origin, gingival or mucosal swelling and status of associated periodontal pockets of teeth needing retreatment) were recorded. The type (extracoronal or intracoronal) and quality (satisfactory or unsatisfactory) of final restorations were assessed according to criteria adapted from the 'Modified USPHS' ${ }^{10}$ Crowns and onlays, either alone or as a bridge abutment, were recorded as extracoronal restorations, whereas direct restorations of silver amalgam or composite resin and all indirect inlays were recorded as intracoronal restorations. Restorations were regarded as unsatisfactory if there were any secondary carious lesions, marginal defects that the explorer could penetrate, or any fracture or loss of the restoration. We assessed the quality of root canal obturation by examining periapical radiographs ${ }^{11}$. Obturation was considered unsatisfactory if (i) the end of the obturation filling was more than $2 \mathrm{~mm}$ short of the apex or had extruded beyond the apex; and (ii) space was visible laterally along the obturated canal or voids were present within the filling mass. Obturation was considered satisfactory if (i) there was uniform radiodensity and adaptation of the filling to the root canal walls; and (ii) the root filling ended short of the apex by $2 \mathrm{~mm}$ or less. All the clinical assessment was accomplished by two investigators together at the same time.

\section{Bacterial sampling}

Samples were collected from both saliva and root canals of each patient and cultured for bacteria. Saliva samples were collected by the method described by Sedgley. ${ }^{6}$ Patients rinsed their mouths for $60 \mathrm{~s}$ with $10 \mathrm{~mL}$ of sterile distilled water and transferred the sample to a $50-\mathrm{mL}$ polypropylene tube, which was stored at $4{ }^{\circ} \mathrm{C}$ and processed in the laboratory within 2 hours. Root canals were swabbed by using the method described by Gomes et al. ${ }^{12}$. The affected tooth was isolated with a rubber dam and disinfected with 5.25\% sodium hypochlorite, which was inactivated with $5 \%$ sodium thiosulfate. Aseptic techniques were followed throughout endodontic therapy and sampling. When the previous restoration was removed and root canal orifice was loacted, the pulp chamber was disinfected with $5.25 \%$ sodium hypochlorite and the previous obturation was removed with Protaper nickeltitanium rotary instruments S1-F2 (Dentsply Tulsa Dental, Tulsa, OK, USA) under irrigation with sterile physiological saline solution. Canal patency was established with minimal instrumentation. Sterile saline was then used to wet the canal and a microbial sample was taken by inserting three sterile paper points into the full length of the canal and keeping them in place for $30 \mathrm{~s}$. The debris located in the apical third of the root canal and the paper points were placed into a 2-mL centrifuge tube containing $1.5 \mathrm{~mL}$ viability medium Gotenberg agar III transport medium and samples were immediately assessed.

\section{Laboratory assessment}

Oral rinse samples were centrifuged at $4{ }^{\circ} \mathrm{C}$ for $10 \mathrm{~min}$ at $13000 \mathrm{~g}$ and pellets were resuspended in $1 \mathrm{~mL}$ of sterile nuclease-free water (Invitrogen, Carlsbad, CA, USA). Meanwhile, centrifuge tubes containing root-canal samples were shaken thoroughly in a mixer for $60 \mathrm{~s}$, and $\log -10$ serial dilutions were made with viability medium Gotenberg agar III transport medium. With the use of a spiral plater (Model D; Spiral Systems Inc, Cincinnati, OH, USA), a $50-\mu \mathrm{L}$ inoculum of each prepared sample was plated onto brain-heart infusion agar plates and bile esculinazide agar plates to culture all bacteria and to select for enterococci, respectively. Plates were incubated aerobically for $48 \mathrm{~h}$ at $37^{\circ} \mathrm{C}$. Colonies that were presumptively identified as enterococci based on bile esculinazide hydrolysis were purified by streak-plating onto a fresh esculinazide agar plate. Isolates were characterized as catalase-negative, non-motile, Gram-positive cocci if they were capable of growth in Todd Hewitt agar (Difco; Becton, Dickinson \& Company, Sparks, NV, USA) supplemented with $6.5 \%$ sodium chloride at $42{ }^{\circ} \mathrm{C}$. They were then identified as E. faecalis with Analytical Profile Index 20 Strep identification kits (Bio Mérieux SA, Marcy-l'Etoile, France) and thel6-S rRNA technique, ${ }^{13}$ with type strain E. faecalis ATCC 29212 serving as a positive control.

To confirm the selective culture results, the E. faecalis-specific polymerase chain reaction (PCR) primer was prepared according to the method of Sedgley. ${ }^{14}$ Universal bacterial primer positive control and E. faecalis-specific primer positive control were used in the procedure. The $25-\mu \mathrm{L}$ reaction mixtures contained $0.5 \mu \mathrm{L}$ of primer, $2.5 \mu \mathrm{L}$ of $10 \times$ PCR buffer, $0.5 \mu \mathrm{L}$ Taq DNA polymerase (Gibco BRL, Gaithersburg, MD, USA) and $0.5 \mu \mathrm{L}$ deoxyribonucleoside triphosphates. The PCR protocol comprised an initial denaturation step at $95{ }^{\circ} \mathrm{C}$ for $15 \mathrm{~min}$, followed by 35 cycles of a denaturation step at $94{ }^{\circ} \mathrm{C}$ for $20 \mathrm{~s}$, a primer annealing step at $50{ }^{\circ} \mathrm{C}$ for $45 \mathrm{~s}$, an extension step at $72{ }^{\circ} \mathrm{C}$ for $30 \mathrm{~s}$ and a final step of $72{ }^{\circ} \mathrm{C}$ for $5 \mathrm{~min}$. Amplicons were analyzed by $1.5 \%$ agarose gel electrophoresis performed at $4 \mathrm{~V} \cdot \mathrm{cm}^{-1}$ in Tris-borate EDTA buffer. The gel was stained with $0.5 \mathrm{mg} \cdot \mathrm{mL}^{-1}$ ethidium bromide and photographed under ultraviolet light (Figure 1).

\section{Statistical analysis}

The data were analyzed with SPSS 17.0 (SPSS Inc., Chicago, IL, USA). In the bivariate analysis, Fisher's exact test was used to assess the statistical significance of the association between the presence of $E$. faecalis in root canals and its presence in saliva, tooth location (anterior or posterior), number of root canals (single or multiple),

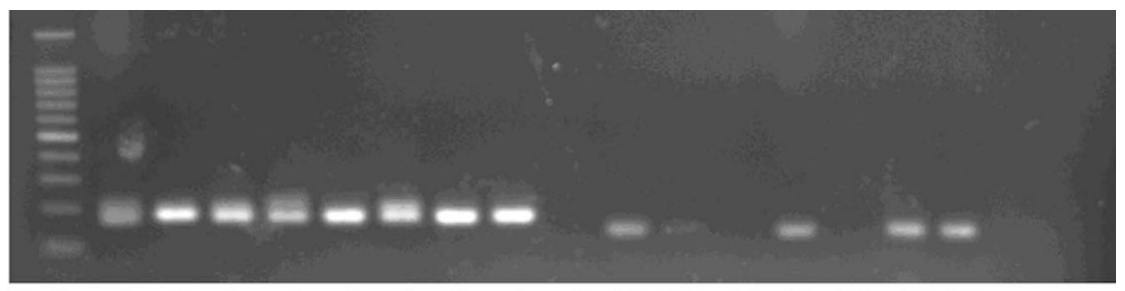

$\begin{array}{lllllllllllllllll}\text { Marker } & 1 & 2 & 3 & 4 & 5 & 6 & 7 & 8 & 9 & 10 & 11 & 12 & 13 & 14 & 15 & 16\end{array}$

Figure 1 Identification with 16S-rRNA technique. Lane 1, universal bacterial primer negative control; lane 2, universal bacterial primer positive control; lanes 3-8, transcription amplification of microbial samples; lane 9, E. faecalis specific primer negative control; lane 10, E. faecalis specific primer positive control; lanes 11-16, transcription amplification of microbial samples. 
Table 1 Presence of $E$. faecalis in root canal, by selected characteristics

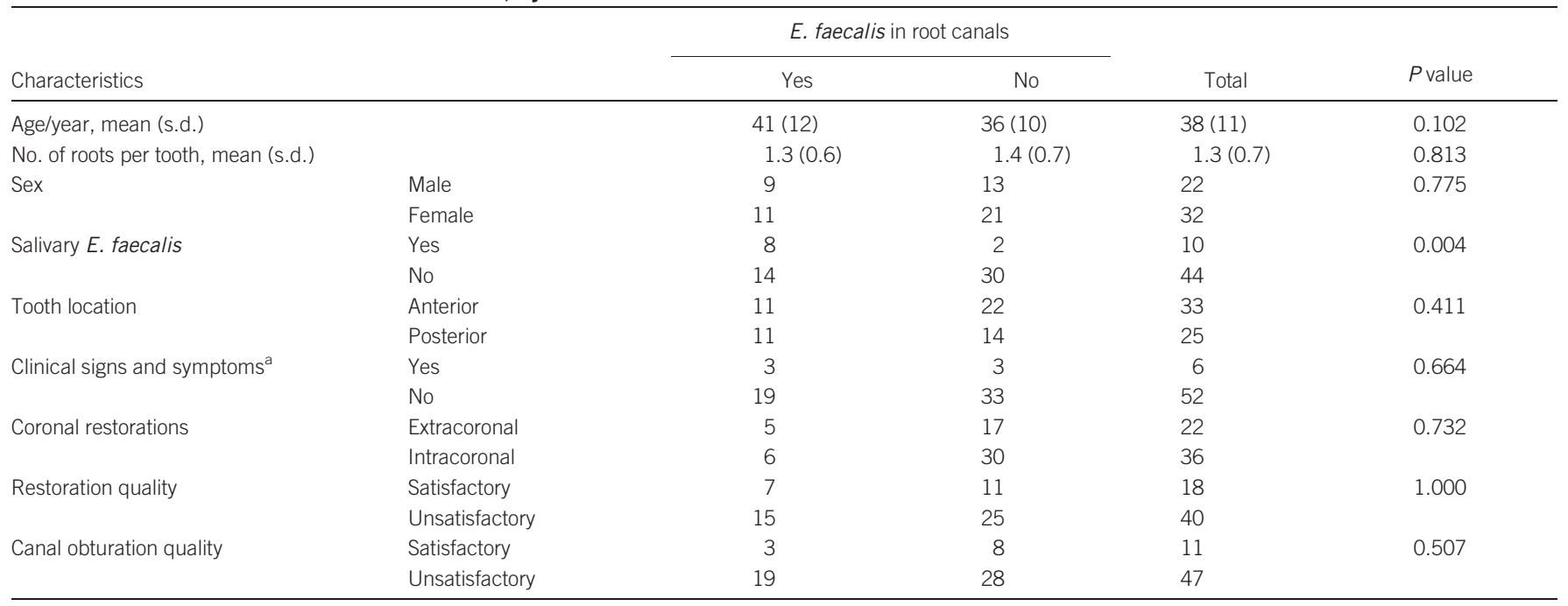

a Signs and symptoms included presence of pain, hypermobility, sinus tract and its origin, gingival and mucosal swelling, and associated periodontal pockets.

presence of clinical signs and symptoms which were mentioned above (yes or no), type of coronal restoration (extracoronal or intracoronal), restoration quality (satisfactory or unsatisfactory) and quality of canal obturation (satisfactory or unsatisfactory). The Chi-square test was performed if at least $80 \%$ of cells had an expected frequency of at least 5 and no cell had an expected frequency of less than 1 . The $t$-test was used to analyze whether age and number of roots differed according to the presence or absence of E. faecalis in root canals, and whether the number of bacterial species cultured from the root canal differed according to the obturation quality. The data will be assessed for normal distribution properties using the Shapiro-Wilk test for normality. Logistic regression analysis was conducted to assess the effects of the above independent variables in a multivariate model in which the presence of E. faecalis in root canals $(1=y e s ; 2=$ no) was the independent variable. The cutoff point for statistical significance was set at 0.05 .

\section{RESULTS}

Fifty-four patients, 32 men and 22 women, who required endodontic retreatment, were recruited in this study. Their ages ranged from 18 to 70 years and their mean age was 38 years (s.d.: 11 years). A total of 58 teeth-29 incisors, 4 canines, 19 premolars, and 6 molars-were studied. In the present study, there were $6.8 \%$ of teeth $(4 / 58)$ with negative culture on the plates.

The prevalence of E. faecalis was 19\% (10 in 54 patients) in saliva and $38 \%$ (22 in 58 teeth) in root canals. A statistically significant association $(P<0.05)$ was found between the presence of $E$. faecalis in saliva (10/54) and its presence in root canals (22/58) (Table 1). Only the presence of E. faecalis in saliva remained significantly associated with increased odds of identifying E. faecalis in root canals (odds ratio $=9.7 ; 95 \%$ confidence interval $=1.8-51.6 ; P<0.05)$ (Table 2). The Cox and Snell $R^{2}$ value was 0.144 .

The tooth locations, presence of clinical symptoms, type of coronal restoration, restoration status, number of root canals and quality of canal obturation were not significantly associated with the presence of E. faecalis in root canals. These findings were confirmed in the logistic regression analysis, so these variables were removed from the final model.
Using our culture method, we found up to four identifiable species of bacteria in their roots (Table 3). The overall mean number of bacterial species identified was 2.3 (s.d.: 1.1) (Table 4). All enterococcal isolates were identified as E. faecalis. We found that 17 teeth had a single identifiable species of bacteria in their roots. Of these 17 teeth, 5 harbored E. faecalis. Among the teeth that required retreatment, those showing satisfactory canal obturation were more likely to harbor only a single cultivable species of bacteria $(P<0.05)$ and had a lower mean number of cultivable bacterial species $(P<0.05)$ than those showing unsatisfactory obturation.

Table 2 Final logistic regression model of $E$. faecalis in root canals

\begin{tabular}{lccc}
\hline Factors & $\beta$ (s.e.) & Odds ratio $(95 \% \mathrm{Cl})$ & $P$ value \\
\hline Presence of E. faecalis in saliva & & & \\
Yes & $-2.274(0.852)$ & $9.7(1.8-51.6)$ & 0.003 \\
$N^{N^{a}}$ & $0.887(0.318)$ & & 0.005 \\
Constant & & 0.005 \\
\hline
\end{tabular}

${ }^{\text {a }}$ Reference category.

Table 3 No. of identifiable species of bacteria and the presence of E. faecalis in the root canals

\begin{tabular}{lrrrrr}
\hline & \multicolumn{4}{c}{ No. of identifiable species of bacteria } & \\
\cline { 2 - 5 } E. faecalis & 1 & 2 & 3 & 4 & Total \\
\hline Yes & 5 & 6 & 6 & 3 & $20(37 \%)$ \\
No & 12 & 4 & 14 & 4 & $34(63 \%)$ \\
Total & 17 & 10 & 20 & 7 & $54(100 \%)$ \\
\hline
\end{tabular}

Table 4 Bacterial profile in non-selective medium according to quality of root canal obturation

\begin{tabular}{lcccc}
\hline & \multicolumn{3}{c}{ Root canal obturation } & \\
\cline { 2 - 4 } Bacteria identified & Satisfactory & Unsatisfactory & All & $P$ value \\
\hline Mean no. (s.d.) & $1.1(0.3)$ & $2.6(1.0)$ & $2.3(1.1)$ & $<0.05$ \\
$\%$ teeth with only one species & $89 \%$ & $20 \%$ & $32 \%$ & $<0.05$ \\
\hline
\end{tabular}




\section{DISCUSSION}

In the present study, E. faecalis could be found in root canals of teeth with apical periodontitis requiring endodontic retreatment or in the saliva, or in both. The prevalence of E. faecalis in root canals and saliva was $38 \%$ and $19 \%$, respectively. These results agree with those of other studies. For example, the reported prevalence of E. faecalis in root canals ranges from $32 \%$ to $70 \%$, with $E$. faecalis being the predominant species in filled root canals with persistent apical periodontitis. ${ }^{12,15-17}$ The reported prevalence of E. faecalis in oral saliva of endodontic patients is lower, at $10 \%-17 \% .{ }^{5-6}$ In contrast, an early study reported that $75 \%$ of endodontic patients had detectable Enterococcus species in saliva. ${ }^{18}$ The conflicting results indicates more clinical studies should be performed to test the hypothesis that oral cavity provides an available source of $E$. faecalis to enter root canals.

Although E. faecalis was detected less frequently in oral rinse (10\%) than from tongue (42\%) and gingival sulcus $(14 \%),{ }^{5}$ it is more likely that $E$. faecalis from oral rinse stays in a planktonic situation, and gets more chance to enter the root canal system than that from tongue or gingival sulcus with a biomembrane structure. We focused on the planktonic E. faecalis in oral cavity in this study.

The most obvious source of E. faecalis in failed root canals is thought to be the oral cavity. However, our findings suggest the prevalence of E. faecalis in saliva of patients undergoing endodontic retreatment is low. Since most root canal samples that were positive for E. faecalis corresponded to saliva that were also positive, there could an association between the presence of E. faecalis in root canals and its presence in saliva. We did not include healthy controls for the saliva tests, but the literature generally suggests that E. faecalis is also not a common oral colonizer in oral cavities with healthy dentition. No detectable enterococci were found in the saliva of dentate people who had never received endodontic treatment. ${ }^{6,8}$

It should be noted that cross-sectional studies are limited, being single views of a microflora existing in a complex dynamic environment and not reproducible within the same individual on different occasions. Future studies could examine the prevalence and characteristics of enterococci recovered in longitudinal studies. If a transient oral infection with E. faecalis occurred during or after treatment, the bacteria might enter the unsealed root canal. If the conditions of root canals favor the survival of enterococci, a long-standing local infection may become established. ${ }^{8}$ Still, the oral cavity seems to be the most likely source of E. faecalis in failed root canal treatment. A laboratory study showed that E. faecalis in the starvation phase could develop a biofilm on human dentin. E. faecalis in a biofilm has much more resistance to $5.25 \%$ sodium hypochorite than stationary cells, and resistance increased as the biofilm matured. Such a mechanism may contribute to the predominant role of E. faecalis in persistent periapical infections, ${ }^{19}$ but unanswered questions include when and how the bacteria enter filled root canals, and what variables in primary endodontic treatment may make the root canal microenvironment conducive to bacterial growth.

The quality of obturation often reflects the quality of endodontic treatment; in addition, roots which are clean and of proper shape are generally easy to obturate satisfactorily. In this study, the microflora of unsatisfactorily obturated root canals was mixed, whereas that of satisfactorily obturated root canals harbored only one species based on the culture technique, probably owing to the lack of space or nutrients to support more than one type of microorganism. In addition, the culture-based technique would impact the biodiversity found which was mainly discussed in the latter part. Our findings agree with those of other researchers. For example, Gomes found that obturated teeth generally harbored one to two species, with facultative anaerobic and Gram-positive bacteria predominating. ${ }^{12}$ Sundqvist reported a similar result and suggested that the number of species isolated from retreatment cases was probably contingent on the quality of the initial endodontic treatment. ${ }^{20}$ The results of this study also agree with Sundqvist that teeth with inadequate endodontic treatment are more likely than teeth with apparently well-cleaned canals to have a microflora similar to that found in untreated canals, and also more likely to contain a greater number of species. Studies reported that the increased occurrence of Gram-positive bacteria in particular may be a consequence of their strong resistance to instrumentation and antiseptic agents. ${ }^{13,20}$

We found that E. faecalis was more common in root canals showing unsatisfactory obturation than in those showing satisfactory obturation (19/58 vs. 3/58), but the prevalence was not significantly associated with the quality of obturation. Unsatisfactorily obturated root canals could provide more space and nutrition than well-obturated canals, and the available space may create a facultative anaerobic environment. In contrast, well-obturated canals maintain an obligate anaerobic environment that does not favor the survival and growth of E. faecalis. Inadequate cleaning and shaping may also have left infected debris behind. Microorganisms such as E. faecalis can survive within the small canals of apical ramifications or in the space between the root filling and canal wall. In fact, E. faecalis strains can survive for at least 6-12 months in an environment where nutrients are scant and when commensality with other bacteria is reduced. ${ }^{6-7}$ E. faecalis is also extremely resistant to chemicals, including calcium hydroxide. ${ }^{21-23}$

E. faecalis has been commonly located in the apical third of the root canal, suggesting that invasion might have occurred during endodontic treatment. ${ }^{24}$ It has also been suggested that a positive association may exist between the occurrence of E. faecalis and the number of clinic visits, owing to coronal microleakage through the temporary filling placed between endodontic treatment sessions. ${ }^{25}$ During the coronal restoration phase, obturated root canals may become exposed to the oral cavity at some point during treatment, especially when temporary restoration is required during the construction of an indirect restoration. Thus, the quality of the temporary restoration may be important to the prevalence of E. faecalis infection in root canals. ${ }^{26}$

A study found E. faecalis was resistant to removal by root canal preparation followed by intracanal dressing. ${ }^{27}$ E. faecalis has been found more frequently in filled canals without a radiographic lesion than in those with a lesion, suggesting that bacterial entry can happen after obturation. ${ }^{9}$ On the other hand, extracoronal restoration with a satisfactory margin can reduce the risk of post-treatment extracoronal leakage and increase the success rate of root canal therapy. ${ }^{28}$ In this study, however, we did not find any association between restoration quality or type and prevalence of E. faecalis in root canals or saliva. This result could be due to the low sensitivity of using Fisher's exact test on small samples.

It should be noted that our culture technique is commonly used for assessing E. faecalis in saliva. ${ }^{29}$ Furthermore, to confirm the identity of E. faecalis, we included a molecular method of PCR. Researchers using molecular methods have recently demonstrated that the microflora associated with endodontic infections is more diverse than that reported by researchers using only conventional culture methods and PCR was more sensitive than culturing in the detection of $E$. faecalis. ${ }^{30}$ But the culture and Analytical Profile Index methods could be used to detect the activity of the isolated E. faecalis. Combining molecular and culture technique is probably the best approach available to provide comprehensive information about the microflora 
associated with endodontic infections. As an illustration of this point, E. faecalis had been isolated in fissure caries using a conventional culture technique, at three orders of magnitude below counts of Streptococcus mutans. ${ }^{31}$ With the use of modern culture and molecular identification techniques, however, enterococci could not be found in carious dentin. ${ }^{32}$ Thus, it seems unlikely that enterococci could occur in carious lesions and act as a source of E. faecalis in root canals.

In the present study, there were $6.8 \%$ of teeth (4/58) with negative culture on the plates. Earlier studies were unable to isolate bacteria from $20 \%,{ }^{16} 15 \%{ }^{15}$ and $10 \%{ }^{12}$ of teeth. However, failure to detect bacteria does not prove their absence; it is possible that some microorganisms could have been lost, especially if the number of microorganisms present in the root canal was very low or microorganisms were the obligate anaerobes. ${ }^{15}$ This information should need further studies to figure out.

In conclusion, we have shown here that the prevalence of $E$. faecalis in root canals is associated with the presence of E. faecalis in saliva. Besides thorough root canal cleaning, shapings and obturation, maintaining hermetic coronal sealing during and after root canal treatment is essential to prevent E. faecalis contamination and colonization of root canals. It is clear that further, larger studies are needed to definitively support or challenge the theory that enterococci can enter the root canal system during or after root canal treatment.

\section{ACKNOWLEDGEMENTS}

This study was supported by the National Natural Science Foundation of China (Grant Nos. 30840091 and 81000428). The authors would like to thank Dr Trevor Lane for editing a draft of this manuscript.

1 Kayaoglu G, Orstavik D. Virulence factors of Enterococcus faecalis: relationship to endodontic disease. Crit Rev Oral Biol Med 2004; 15(5): 308-320.

2 Souto R, Colombo AP. Prevalence of Enterococcus faecalis in subgingival biofilm and saliva of subjects with chronic periodontal infection. Arch Oral Biol 2008; 53(2): $155-160$.

3 Love RM. Enterococcus faecalis - a mechanism for its role in endodontic failure. Int Endod J 2001; 34(5): 399-405.

4 Sunde PT, Olsen I, Debelian GJ et al. Microbiota of periapical lesions refractory to endodontic therapy. J Endod 2002; 28(4): 304-310.

5 Sedgley C, Buck G, Appelbe O. Prevalence of Enterococcus faecalis at multiple oral sites in endodontic patients using culture and PCR. J Endod 2006; 32(2): 104-109.

6 Sedgley CM, Lennan SL, Clewell DB. Prevalence, phenotype and genotype of oral enterococci. Oral Microbiol Immunol 2004; 19(2): 95-101.

7 Kampfer J, Gohring TN, Attin T et al. Leakage of food-borne Enterococcus faecalis through temporary fillings in a simulated oral environment. Int Endod J 2007; 40(6): 471-477.

8 Razavi A, Gmur R, Imfeld T et al. Recovery of Enterococcus faecalis from cheese in the oral cavity of healthy subjects. Oral Microbiol Immunol 2007; 22(4): 248-251.

9 Kaufman B, Spangberg L, Barry J et al. Enterococcus spp. in endodontically treated teeth with and without periradicular lesions. J Endod 2005; 31(12): 851-856.

10 Bayne SC, Schmalz G. Reprinting the classic article on USPHS evaluation methods for measuring the clinical research performance of restorative materials. Clin Oral Investig 2005; 9(4): 209-214.
11 Dugas NN, Lawrence HP, Teplitsky PE et al. Periapical health and treatment quality assessment of root-filled teeth in two Canadian populations. Int Endod J 2003; 36(3): 181-192.

12 Gomes BP, Pinheiro ET, Gade-Neto CR et al. Microbiological examination of infected dental root canals. Oral Microbiol Immunol 2004; 19(2): 71-76.

13 Schirrmeister JF, Liebenow AL, Pelz K et al. New bacterial compositions in root-filled teeth with periradicular lesions. J Endod 2009; 35(2): 169-174.

14 Sedgley CM, Nagel AC, Shelburne CE et al. Quantitative real-time PCR detection of oral Enterococcus faecalis in humans. Arch Oral Biol 2005; 50(6): 575-583.

15 Pinheiro ET, Gomes BP, Ferraz CC et al. Microorganisms from canals of root-filled teeth with periapical lesions. Int Endod J 2003; 36(1): 1-11.

16 Peciuliene V, Balciuniene I, Eriksen HM et al. Isolation of Enterococcus faecalis in previously root-filled canals in a Lithuanian population. J Endod 2000; 26(10): 593595.

17 Adib V, Spratt D, Ng YL et al. Cultivable microbial flora associated with persistent periapical disease and coronal leakage after root canal treatment: a preliminary study. Int Endod J 2004; 37(8): 542-551.

18 Gold OG, Jordan HV, van Houte J. The prevalence of enterococci in the human mouth and their pathogenicity in animal models. Arch Oral Biol 1975; 20(7): 473-477.

19 Liu H, Wei X, Ling J et al. Biofilm formation capability of Enterococcus faecalis cells in starvation phase and its susceptibility to sodium hypochlorite. J Endod 2010; 36(4): 630-635.

20 Sundqvist G, Figdor D, Persson S et al. Microbiologic analysis of teeth with failed endodontic treatment and the outcome of conservative re-treatment. Oral Surg Oral Med Oral Pathol Oral Radiol Endod 1998; 85(1): 86-93.

21 Evans M, Davies JK, Sundqvist G et al. Mechanisms involved in the resistance of Enterococcus faecalis to calcium hydroxide. Int Endod J 2002; 35(3): 221-228.

22 Portenier I, Haapasalo H, Orstavik D et al. Inactivation of the antibacterial activity of iodine potassium iodide and chlorhexidine digluconate against Enterococcus faecalis by dentin, dentin matrix, type-I collagen, and heat-killed microbial whole cells. $J$ Endod 2002; 28(9): 634-637.

23 Dahlen G, Samuelsson W, Molander A et al. Identification and antimicrobial susceptibility of enterococci isolated from the root canal. Oral Microbiol Immunol 2000; 15(5): 309-312.

24 Nair PN, Henry S, Cano V et al. Microbial status of apical root canal system of human mandibular first molars with primary apical periodontitis after "one-visit" endodontic treatment. Oral Surg Oral Med Oral Pathol Oral Radiol Endod 2005; 99(2): 231-252.

25 Siren EK, Haapasalo MP, Ranta K et al. Microbiological findings and clinical treatment procedures in endodontic cases selected for microbiological investigation. Int Endod J 1997; 30(2): 91-95.

26 Zehnder M, Guggenheim B. The mysterious appearance of enterococci in filled root canals. Int Endod J 2009; 42(4): 277-287.

27 Ferrari PH, Cai S, Bombana AC. Effect of endodontic procedures on enterococci, enteric bacteria and yeasts in primary endodontic infections. Int Endod J 2005; 38(6): 372-380.

$28 \mathrm{Chu} \mathrm{CH}$, Lo EC, Cheung GS. Outcome of root canal treatment using Thermafil and cold lateral condensation filling techniques. Int Endod J 2005; 38(3): 179-185.

29 Samaranayake LP, MacFarlane TW, Lamey PJ et al. A comparison of oral rinse and imprint sampling techniques for the detection of yeast, coliform and Staphylococcus aureus carriage in the oral cavity. J Oral Pathol 1986; 15(7): 386-388.

30 Siqueira JF Jr, Rocas IN. Pseudoramibacter alactolyticus in primary endodontic infections. J Endod 2003; 29(11): 735-738.

31 Meiers JC, Wirthlin MR, Shklair IL. A microbiological analysis of human early carious and non-carious fissures. J Dent Res 1982; 61(3): 460-464.

32 Martin FE, Nadkarni MA, Jacques NA et al. Quantitative microbiological study of human carious dentine by culture and real-time PCR: association of anaerobes with histopathological changes in chronic pulpitis. J Clin Microbiol 2002; 40(5): 16981704.

\section{(c)}

This work is licensed under a Creative Commons Attribution-NonCommercial-NoDerivative Works 3.0 Unported License. To view a copy of this license, visit http:// creativecommons.org/licenses/by-nc-nd/3.0 